\title{
Childbirth as a trauma: Psychometric properties of the impact event scale in Japanese mothers of neonates
}

\author{
Mizuki Takegata ${ }^{1,2,{ }^{*}, \text { Toshinori Kitamura }}{ }^{2,3}$, Megumi Haruna ${ }^{1}$, Kyoko Sakanashi ${ }^{4}$, \\ Tomoko Tanaka ${ }^{5}$ \\ ${ }^{1}$ Department of Midwifery and Women's Health, the University of Tokyo, Tokyo, Japan \\ ${ }^{2}$ Kitamura Institute of Mental Health Tokyo, Tokyo, Japan \\ ${ }^{3}$ Department of Psychiatry, Nagoya University, Nagoya, Japan \\ ${ }^{4}$ Department of Women's Health/Mother-Child Nursing, Faculty of Life Science, Kumamoto University, Kumamoto, Japan \\ ${ }^{5}$ Aso Public Health Center, Kumamoto, Japan
}

\section{Email address:}

mtakegata-tky@umin.ac.jp (M. Takegata)

\section{To site this article:}

MizukiTakegata, Toshinori Kitamura, Megumi Haruna, Kyoko Sakanashi, Tomoko Tanaka. Childbirth as a Trauma: Psychometric Properties of the Impact Event Scale in Japanese Mothers of Neonates. Psychology and Behavioral Sciences. Vol. 3, No. 2, 2014, pp. 46-50. doi: 10.11648/j.pbs.20140302.12

\begin{abstract}
Introduction:The purpose of this study was to evaluate the psychometric properties of the Impact of Event Scale (IES) among Japanese women after childbirth. Methods: Self-reported questionnaires were distributed to pregnant women in Kumamoto prefectureconsecutivelyon three occasions: late pregnancy $(\mathrm{N}=642)$ andfive days $(\mathrm{N}=416)$ and one month $(\mathrm{N}=$ 226) after delivery. Results:An exploratory factor analysis of the IES items at day 5 after childbirth yielded a two-factor structure (Intrusion and Avoidance) that was cross-validated by a confirmatory factor analysis. The IES subscale scores were correlated with other psychological measures rated at the same time, including postpartum depressive symptoms assessed with the Edinburgh Postnatal Depression Scale (EPDS) and perceived difficulty of childbirth experience. The Cronbach'salphacoefficients were .83 for Intrusion and .86 for Avoidance. Test-retest reliability between day 5 and one month after childbirth was .74 for Intrusion and .71 for Avoidance. Discussion: The results provide support for the IES as a valid and reliable measure of postnatal traumatic symptoms among Japanese women.
\end{abstract}

Keywords:Impact of Event Scale, Japanese, Postnatal Women, Translation, Traumatic Stress

\section{Introduction}

Childbirth can be a life-threatening event for both mothers and infants, with unpredictable risks. Some women see their childbirth as a traumatic event (1). In the United Kingdom and Nordic countries, it has been reported that approximately $1-7 \%$ of postnatal women fulfil the criteria for post-traumatic stress disorder (PTSD) following childbirth, and a larger number of postpartum women (24-34\%) may have one or more traumatic stress symptoms(1- 4). Several contributors to traumatic stress symptoms following childbirth have been identified:anindividual's vulnerability to stressful situations such as anxiety disorders and depressive symptoms during pregnancy(5); prolonged or invasive obstetrical procedures such as an emergency Caesarean section (emCS), or a forceps or a vacuum delivery (2); and perceived lack of support from health care professionals during delivery
$(2,3,6)$. Traumatic stress following childbirth may have adverse consequences over both short-andlong-term periods. Traumatic birth can interfere with a mother's attachment to her infant and her overall adjustment $(7,8)$, as well as negatively affect therelationship with her partner $(8,9)$ and her decisions about having additional children $(10,11)$.

We are aware of only one report related to traumatic stress following childbirth in Japan (12). Conducting semi-structured interviews to investigate PTSD criteria (DSM-IV) at one month after delivery, the above study found that 8 of 11 women who delivered by emCShad one or more traumatic stress symptoms. However, the number of participants in that study was small. Minimal research regarding traumatic stress symptoms following childbirth has been performed in a larger population in Japan. This may be partly due to the unavailability of suitable scalesthat have proved valid and reliable in Japanese population.

We considered that the Impact of Event Scale (IES)(13), a 
widely used self-report of traumatic symptoms, might be useful as a measure of birth-related traumatic symptoms. The IES consists of 15 items. The two-dimensional factor structure was identified in the English version in postnatal women following traumatic birth experiences (14): Intrusion and Avoidance. In Avoidance, an individual avoids talking or reading about trauma-related issues, whereas Intrusion refers to re-experiencing, nightmares, and flashbacks.

The aims of this study were to translate the IES into Japanese and to examine its psychometric properties in Japanese mothers of neonates.

\section{Methods}

\subsection{Translation and Linguistic Validation}

The original IES was translated into Japanese by one of us (T.K.) with the permission of the original author. The translated version was then reviewed for its intelligibility and the proper wordings in a Japanese cultural context by several Japanese-speaking experts (including us) with backgrounds in psychiatry and psychology. If recommended, phrases were re-translated from English to Japanese. Then, the Japanese version was translated back into English by two other independent bilingual translators. The original and the back-translated versions were compared by the researchers in order to determine the clarity of the translation work and the semantic and content equivalence of the different language versions of the questionnaire.

\subsection{Psychometric Study}

\subsubsection{Participants}

The Kumamoto Prefectural Government solicited all 55 obstetric clinics in Kumamoto Prefecture to participate in this follow-up survey of mental health during the perinatal period in community pregnant women. Eighteenantenatal institutes (33\%) responded. These included one university hospital, public and private hospitals $(\mathrm{N}=12)$, and private clinics $(\mathrm{N}=5)$. Thus, we obtained a mixture of different types of antenatal institutions, which were astrength of the current study. Women of at least 28 weeks' gestation who attended one of these antenatal clinics during the entire month of November 2011 were invited to participate. Eligible criteria were pregnancyand age over 20 years. Pregnant women who were illiterate in Japanese, who had severe mental illness, or who had been hospitalized with pregnancy complications were excluded. Sets of questionnaires were distributed during the third trimester of pregnancy, and at five days and one month after childbirth. Of 1442 eligible women, 642(45\%) returned the questionnairesduring the third trimester and 416 (29\%) returned them within five days after childbirth. Of those who returned the questionnaires at one month after childbirth, $226(16 \%)$ mothers returned them one month after childbirth.

\subsubsection{Measurement}

\section{Traumatic symptoms}

The IES (13) is a self-report of traumatic stress symptoms. It consists of 15 items with two dimensions (Intrusion: seven items, and Avoidance: eight items), rated on a five-point scale, ranging from "not at all (0)" to "quite often (4)."Higher scores indicate a severe traumatic stress symptoms. In this study, we asked "How was your childbirth experience?" This question was followed by the 15-IES items. The IES was measured at five days after childbirth (N $=416)$, and again at one month after delivery $(\mathrm{N}=226)$ in order to examine its test-retest reliability.

Depression

Symptoms of maternal depression were assessed within five days after delivery by the Edinburgh Postnatal Depression Scale (EPDS)(15), a screening instrument for postpartum depression. The scale consists of 10 items with a four-point scale. The EPDS was translated into Japanese by Okano et al(16), who examined its validity and reliability. We hypothesized that there would be a moderate correlation between the IES and the EPDS scores(17). The Cronbach's alpha value of the EPDS score was .84 in this study.

Perception of difficulty of childbirth

An ad hoc question, "How was your childbirth experience?" was included in the questionnaire. The question was rated on a five-point scale ranging from "very easy (1)" to "very difficult (7)".

Demographic and obstetric data

Demographic data included age and parity (primiparous/multiparous). Medical records were used to identify complications of pregnancyincluding threatened labor, pregnancy hypertension, placenta previa,and anomalies of the foetus, and birth outcomes including planned CS, emCS, and other delivery complications.

\subsubsection{Data Analyses}

In order to examine the factor structure of the IES, we randomly divided the participants into two groups and performed an exploratory factor analysis (EFA) on the first group. Robust maximum likelihood estimation procedures were employed. The number of factors was determined through the use of scree plots after confirming that the eigenvalues of all retained factors were $>1$. Promax rotation was performed to obtain a diagonal rotation, because all IES subscales were considered somewhat interdependent. A factor loading of .3 was used as the minimum criterion for acceptance, while .4 was used as the robust criterion (18).

In order to confirm the stability of the factor structures obtained from the above EFA, we performed a series of confirmatory factor analyses (CFAs) using another randomly generated subset of participants $(\mathrm{N}=195)$. The fit of each model with the data was examined in terms of chi-squared (CMIN), comparative fit index (CFI), and root mean square error of approximation (RMSEA). According to conventional criteria, a good fit would be indicated by $\mathrm{CMIN} / d f<2$, CFI $>.97$, and RMSEA $<.05$, and an acceptable fit by $\mathrm{CMIN} / d f<3$, CFI $>.95$, and RMSEA $<.08(19)$. 
We added the scores of items belonging to each factor to calculate the IES subscale scores. Then we calculated the internal consistency of each subscale score using Cronbach's $\alpha$ coefficient. A Cronbach's $\alpha$ value of .7 is considered to be the minimum criterion for acceptance (20).

We also correlated IES subscale scores with the EPDS scores at five days after childbirth. APearson product-moment correlation coefficient $(r)$ was designated as weak (.10-.29), moderate (.30-.49), or high $(\geq .50)$ depending on the value of $\mathrm{r}(21)$.

In order to analyze the test-retest reliability of each IES subscale, we compared the scores of each IES subscale at five days and at one month after delivery.Intraclass correlation coefficient (ICC) values of .4 or less were designated as poor, .41-.60as moderate, .61-.80as good, and .81-1.00 as excellent (22).

All the statistical analyses were conducted using SPSS version 20.0 and Amos 20.0.

\subsubsection{Procedure}

Participants were asked to complete the questionnaire at home and to return it to one of the researchers (T.K.) using a stamp-added envelope. The present study was approved by the Ethical Committee of Kumamoto University Graduate School of Life Sciences.

\section{Results}

The average age of the total sample was $30.4(\mathrm{SD}=4.8)$ years, and $119(29 \%)$ of 410 women were nulliparous. Approximately $14 \%$ of women $(\mathrm{N}=56,14 \%)$ had a pregnancy complication such as threatened abortion (delivery) $(\mathrm{N}=53)$, foetal anomaly $(\mathrm{N}=2)$, or pregnancy hypertension $(\mathrm{N}=1)$. Of the 416 participants, $321(77 \%)$ had a normal vaginal delivery, $41(10 \%)$ had planned CS, and $41(10 \%)$ had emCS.

Only six $(1.3 \%)$ of the 416 participants were missing over $60 \%$ of items on the IES. These questionnaires were excluded from analysis in order to maintain the quality of data. Missing items in the remaining cases were imputed by the mean score of each item.

Because about half of the IES items had a skew of 2.0 or more (items 3, 7, 8, 9, 12, 13, and 15), all the items were log-transformed (Table 1). An EFA using the first group ( $\mathrm{N}=$ 215 ) yielded a two-factor structure (Table 1), accounting for a cumulative variance of $48 \%$. The original two factors, Intrusion and Avoidance, were identified in our sample. No item had a loading of less than .3 on both factors except for Item 2,whichwas originally an Avoidance item but which loaded on the Intrusion category in our study.

Table 1.Factor structure of the IES items in a split-half sample $(N=215)$. Two-factor structure was identified after the exploratory factor analysis.

\begin{tabular}{|c|c|c|c|c|c|c|c|}
\hline \multirow{2}{*}{$\begin{array}{l}\text { Item } \\
\text { number }\end{array}$} & \multirow{2}{*}{ IES items } & \multirow{2}{*}{ Mean (SD) } & \multirow{2}{*}{ Skewness } & \multirow{2}{*}{$\begin{array}{l}\text { Skewness after log } \\
\text { transformation }\end{array}$} & \multirow{2}{*}{ Communality } & \multicolumn{2}{|l|}{ Factor } \\
\hline & & & & & & $\mathbf{I}$ & II \\
\hline 1 & I thought about it when I did not mean to. & $2.40(1.02)$ & 0.07 & -0.31 & 0.21 & -0.14 & 0.52 \\
\hline 2 & $\begin{array}{l}\text { I avoided letting myself get upset then } \\
\text { thought about it or was reminded of it. }\end{array}$ & $1.70(0.86)$ & 0.92 & 0.61 & 0.41 & 0.26 & 0.46 \\
\hline 3 & I tried to remove it from memory. & $1.26(0.62)$ & 2.38 & 2.01 & 0.50 & 0.66 & 0.07 \\
\hline 4 & $\begin{array}{l}\text { Had trouble falling asleep or staying asleep, } \\
\text { because of pictures or thoughts about it that came } \\
\text { into my mind. }\end{array}$ & $1.56(0.87)$ & 1.33 & 1.04 & 0.49 & 0.09 & 0.64 \\
\hline 5 & I had waves of strong feelings about it. & $1.95(0.98)$ & 0.68 & 0.28 & 0.55 & -0.07 & 0.78 \\
\hline 6 & I had dreams about it. & $1.57(0.85)$ & 1.35 & 1.01 & 0.23 & 0.13 & 0.39 \\
\hline 7 & I stayed away from reminders of it. & $1.29(0.66)$ & 2.58 & 2.11 & 0.70 & 0.90 & -0.12 \\
\hline 8 & I felt as if it hadn't happened or it wasn't real. & $1.19(0.56)$ & 3.17 & 2.72 & 0.27 & 0.51 & 0.01 \\
\hline 9 & I tried not to talk about it. & $1.09(0.40)$ & 5.25 & 4.53 & 0.40 & 0.62 & 0.01 \\
\hline 10 & Pictures about it popped into my mind. & $1.93(0.91)$ & 0.70 & 0.27 & 0.57 & -0.13 & 0.82 \\
\hline 11 & Other things kept making me think about it. & $1.59(0.87)$ & 1.32 & 0.98 & 0.51 & 0.11 & 0.64 \\
\hline 12 & $\begin{array}{l}\text { I was aware that I still had a lot of feelings about it, } \\
\text { but I didn't deal with them. }\end{array}$ & $1.29(0.66)$ & 2.51 & 2.08 & 0.52 & 0.53 & 0.27 \\
\hline 13 & I tried not to think about it. & $1.27(0.61)$ & 2.47 & 2.04 & 0.65 & 0.86 & -0.11 \\
\hline 14 & Any reminder brought back feelings about it. & $1.70(0.86)$ & 1.03 & 0.66 & 0.28 & 0.12 & 0.45 \\
\hline 15 & My feelings about it were kind of numb. & $1.17(0.53)$ & 3.68 & 3.09 & 0.49 & 0.69 & 0.01 \\
\hline
\end{tabular}

The final CFA model using the second half-sample $(\mathrm{N}=$ 195) showed a good fit of the two-factor model with the data: $\mathrm{CMIN} / d f=2.51, \mathrm{CFI}=.89$, and $\mathrm{RMSEA}=.08$ (Figure 1).

Then we calculated the two subscale scores by adding the scores of items belonging to each factor. The Cronbach's $\alpha$ values of the IES subscales were above $.7: .83$ for Intrusion and .86 for Avoidance. The two subscales were moderately correlated $(\mathrm{r}=.66, p<.01)$.

Intrusion $(\mathrm{r}=.41, p<.001)$ and Avoidance $(\mathrm{r}=.39, p<.001)$ both showed a moderate correlation with the EPDS scores. Intrusion showed a weak correlation with the perceived difficulty of childbirth $(\mathrm{r}=.20, p<.01)$, while Avoidance failed to show a significant correlation $(\mathrm{r}=.13)$.

Satisfactory test-retest reliability of the two subscales was shown by an ICC of .74 $(p<.001)$ for Intrusion and .71 $(p<.001)$ for Avoidance. 


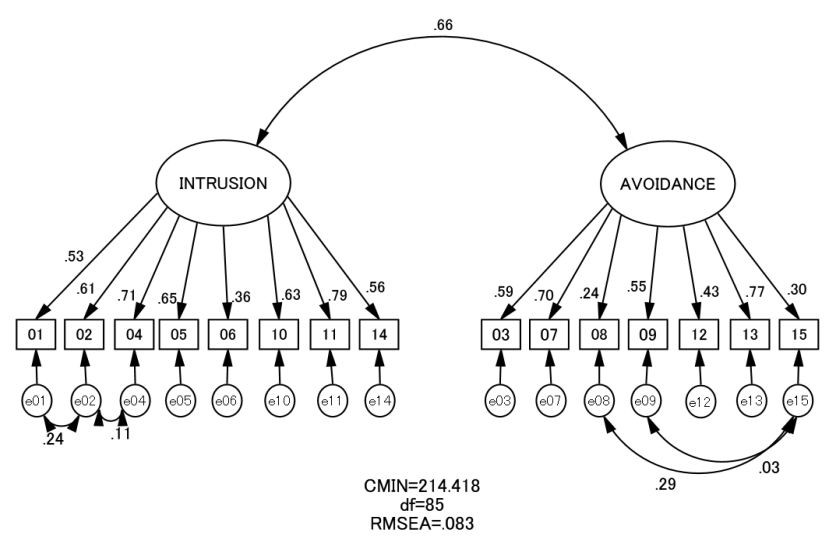

CMIN, chi-squared; df, degree of freedom; RMSEA, root mean square error of approximation; CFI, comparative fit index.

Figure 1.Confirmatory factor analyses of the IES items in a split-half sample $(N=195)$.

\section{Discussion}

The findings of this study provided initial confirmation that the Japanese version of the IES has the same factor structure as the original English one. The instrument's high construct validity and reliability were also demonstrated.

The EFA yielded two factors-Intrusion and Avoidance-in a split-half group. The psychometric structure of the scale showed acceptable fit with the observational data from the results of a CFA performed on the other group. The results confirmed the high construct validity of the Japanese IES and also ensured cross-validation. All items reached the minimum criterion for factor loading $(>.3)$, with most items exhibiting values above .4. These findingsdemonstrate thatJapanese IES items were applicable to Japanese postnatal women. All IES items belonged to the same dimension (Intrusion or Avoidance) as the original items, except for item 2 (original version: "I avoided letting myself get upset when I thought about it or was reminded of it"). This was categorised as belonging to Avoidance in the original English version but loaded highlyon the Intrusion subscale in the present study. When translated into Japanese, this item was expressed as, "When I thought about it or was reminded of it, I avoided letting myself get upset." This is due to a difference in sentence structure between English and Japanese. Therefore, the original English phrase put more emphasis on avoiding behaviour while the Japanese phrase focused attention on intrusion of traumatic thought. Intrusion and avoidance oscillate during the same time period (13). Our finding of moderate correlation between the two IES subscales is inline with this notion $(23,24)$.

An additional finding was that the Japanese IES correlated at expected levels with depressive symptoms, which also supports the fact that its convergent validity is in accordance with a previous study (17). Women who characteristically tend to avoid stressful situationsengage in avoidant behaviours to restore emotional equilibrium and prevent emotional flooding as their defence efforts after traumatic delivery. Our findings provided support for the discriminant and predictable validity of the IES. Only the Intrusion subscale correlated with the perception of difficulty of childbirth. The perceived negative birthexperiences, including strong pain, fear, and lack of control, resulted in development of intrusion symptoms such as re-experiencing and flashbacks.

The IES had good internal consistency and good test-retest reliability at one month after childbirth. The excellent ICC for the total scores of the IES also indicated high stability for the same time frame.

Our study had some limitations. First, the participants were all relatively healthy, pregnant women above 20 years of age. Second, several former researchers recommend the use of the IES-R, which additionally includes a hyper-arousal subscale (25). However, Tham et al (21) claimed that hyper-arousal symptoms such as sleep disturbance are frequently seen among postnatal women. Therefore, we should carefully consider which measures are most suitable for future assessments of traumatic stress symptoms following childbirth. Third, we did not conduct structured interviews to examine the concurrent validity of the Japanese IES. Future studies will be needed to confirm the validation of the scale.

\section{Conclusion}

The results of our study provide support for the Japanese IES as a valid and reliable measure of prenatal traumatic symptoms following childbirth among postnatal Japanese women.

\section{Acknowledgement}

We thank all participating mothers and the staff of the antenatal clinics they attended.

\section{References}

[1] E.Olde,O.Van.Der.Hart,R.Kleber,andM.Van.Son,"Posttraum atic stress following childbirth: A review,"Clin. Psychol. Rev,vol.26, pp.1-16, 2006.

[2] J.Czarnocka, and P.Slade,"Prevalence and predictors of post-traumatic stress symptoms following childbirth,"Br. J. Clin. Psychol, vol.39,pp.35-51, 2000.

[3] D.K. Creedy, I.M. Shochet, and J. Horsfall,"Childbirth and the development of acute trauma symptoms: Incidence and contributing factors,"Birth, vol. 27, pp.104-111, 2000.

[4] S.Ayers, and A.D.Pickering,"Do women get posttraumatic stress disorder as a result of childbirth? A prospective study of incidence,"Birth, vol. 28, pp.111-118, 2002.

[5] S. Ayers, "Commentary on Post-traumatic stress following childbirth: a review of the emerging literature and directions for research and practice," Psychol. Heal. Med, vol. 8, pp. 159-168, 2003. 
[6] S, Ayers,"Assessing psychopathology in pregnancy and postpartum,"J. Psychosom. Obstet. Gynaecol, vol. 22, pp. 91-102, 2001.

[7] C.G. Ballard, A.K. Stanley,I.F. Brockington, "Post-traumatic stress disorder (PTSD) after childbirth," Br. J. Psychiatry, vol. 166, pp.525-528, 1995.

[8] S.Allen,"A qualitative analysis of the process, mediating variables and impact of traumatic childbirth," J. Reprod. Infant Psychol, vol.16, pp.107-131, 1998.

[9] C.T. Beck, "Post-traumatic stress disorder due to childbirth: The aftermath,"Nurs. Res,vol.53, pp. 216-224, 2004.

[10] T.Saisto, K.Salmela-Aro, J.E.Nurmi,T.Könönen, and E. Halmesmäki,"A randomized controlled trial of intervention in fear of childbirth," Obstet. Gynaecol, vol. 98, pp. 820-826, 2001.

[11] E.L.Ryding,A.Persson, C.Onell, and L.Kvist,"An evaluation of midwives' counseling of pregnant women in fear of childbirth," Acta Obstet. Gynecol. Scand.vol. 82, pp. 10-17,2003.

[12] N.Yokote, "Women's experiences of labor, surgery and first postnatal week by an emergency Caesarean section(in Japanese),"J. Japan Acad. Midwifery,vol. 22, pp. 37-48, 2008.

[13] M.Horowitz, N.Wilner, and W.Alvarez,"Impact of Event Scale: A measure of subjective stress,"Psychosom. Med, 41, pp. 209-218,1979.

[14] S.Garthus-Niegel,T.Von.Soest, M.E.Vollrath, andM. Eberhard-Gran,"The impact of subjective birth experiences on post-traumatic stress symptoms: A longitudinal study," ArchWomens Ment Heal, vol. 16, pp. 1-10, 2013.

[15] J.L.Cox, J.M.Holden, and R.Sagovsky,"Detection of postnatal depression. Development of the 10-item Edinburgh Postnatal Depression Scale,"Br. J. Psychiatry,vol.150, pp.782-786.

[16] T.Okano,T.Murata, A.Masuji, A.Tamaki, J.Nomura, and K.
Miyaoka, et al,"Validation and reliability of Japanese version of Edinburgh Postnatal Depression Scale (in Japanese),"Seisinka-Shindangaku. vol, 7, pp. 523-533, 1996.

[17] M.T.Spurrell,andA.C.McFarlane, "Life-events and psychiatric symptoms in a general psychiatry clinic: The role of intrusion and avoidance,"Br. J. Med. Psychol, vol. 68, pp. 333-340.

[18] R.F.DeVellis,Scale development: Theory and applications, 3rded.,New York: Sage Publications, 2003, pp.171.

[19] B.M.Byrne, Structural equation modeling with AMOS: Basic concepts, applications, and programming,Mahwah: Lawrence Erlbaum Associates, 2001, pp. 365-367.

[20] J.C.Nunnally, and I.H.Bernstein, The theory of measurement error. Psychometric Theory,New York: McGraw Hill, 1994, pp. 209-247.

[21] J.Cohen,Statistical power analysis for the behavioral sciences, Mahwah: Lawrence Erlbaum Associates; 1988, pp. 567.

[22] J.J. Bartko, "The intraclass correlation coefficient as a measure of reliability,"Psychol. Report, vol. 19, pp. 3-11,1966.

[23] I.H.Sloan, R.H.Rozensky, L.Kaplan, and S.M.Saunders,"A shooting incident in an elementary school: Effects of worker stress on public safety, mental health, and medical personnel," J. Trauma. Stress, vol. 7, pp. 565-574, 1994.

[24] B.Kelly,B.Raphael, and M.Smithers, etal.,"Psychological responses to malignant melanoma. An investigation of traumatic stress reactions to life-threatening illness. " Gen. Hosp. Psychiatry, vol. 17, pp. 126-134, 1995.

[25] D.S.Weiss, and C.R.Marmar, The Impact of Event Scale Revised.2nd eddition. Assessing Psychological Trauma and PTSD, New York: Guilford Press, 1997, pp.399-411.

[26] Tham V, Christensson K, and Ryding EL. Sense of coherence and symptoms of post-traumatic stress after emergency caesarean section. Acta Obstet. Gynecol. Scand. 2007;86(9):1090-1096. 